\title{
Paired Motor Neuron-Muscle Recordings in Zebrafish Test the Receptor Blockade Model for Shaping Synaptic Current
}

\author{
Hua Wen and Paul Brehm \\ Department of Neurobiology and Behavior, State University of New York at Stony Brook, Stony Brook, New York 11794
}

The transparent spinal cord and electrically compact fast muscle of zebrafish offer the first opportunity to perform simultaneous whole-cell patch-clamp recordings from both motor neuron and target skeletal muscle in situ. Our paired recordings reveal the fastest reported kinetics for both spontaneous and evoked synaptic currents at any synapse and a large quantal size that facilitates the resolution of spontaneous synaptic currents. We used this preparation to test the recent proposal that open channel block of the acetylcholine receptor by acetylcholine modulates the kinetics and timing of transmission between nerve and muscle in larval zebrafish (Legendre et al., 2000). Contrary to the predictions of this model, we find similar delay and onset kinetics of synaptic current at positive and negative muscle membrane potentials, even after inhibition of acetylcholinesterase. In contrast, blockade of motor neuron $\mathrm{K}$ channels by 4-aminopyridine prolonged the action potential and introduced a significant delay and slowing of evoked synaptic currents, demonstrating our ability to measured altered transmitter release with this system. We conclude that the kinetics of neuromuscular synaptic currents in zebrafish is not governed by receptor block.

Key words: neuromuscular; channel block; cholinergic; quantal analysis; patch clamp; spinal cord; K channel

\section{Introduction}

The neuromuscular junction serves as one of the premier models for understanding synaptic transmission. Still, there are no reports in any vertebrate of paired patch-clamp recordings from spinal motor neurons and target skeletal muscle in vivo. In frog and mammals, a major obstacle to patch-clamp measurements of synaptic current is the large size of muscle. Thus, paired patch clamp of spinal neurons and target muscle have been restricted to cultured nerve and muscle, which show limited ability to fully recapitulate the in vivo hallmarks of synapse formation. Therefore, it is critical to record synaptic current responses in vivo, under conditions in which established synapses can be studied. Because of the remarkable transparency of zebrafish, it has been possible to record from the spinal neurons in situ (Saint-Amant and Drapeau, 2003), an approach we now extend to paired recordings from primary motor neurons and target fast muscles. We find that the fast skeletal muscle of the tail is small and electrically compact, facilitating an effective voltage clamp. So, in addition to spontaneous synaptic currents (Nguyen et al., 1999), larval zebrafish now offers a unique opportunity to examine the evoked end-plate current (EPC).

Recently, it was proposed that the kinetics of neuromuscular synaptic transmission in zebrafish muscle is governed by a block of the receptor by ACh (Legendre et al., 2000). According to this

Received June 24, 2005; revised July 26, 2005; accepted July 26, 2005.

This work was supported by National Institutes of Health Grant NS18205. We are grateful to Joe Fetcho and Mark Masino for the help they provided in developing the paired recording technique. We also thank Lonnie Wollmuth and laboratory members for critical comments on this manuscript.

Correspondence should be addressed to Dr. Hua Wen, Department of Neurobiology and Behavior, State University of New York at Stony Brook, Stony Brook, NY 11794. E-mail: hwen@notes.cc.sunysb.edu.

DOI:10.1523/JNEUROSCI.2611-05.2005

Copyright $\odot 2005$ Society for Neuroscience $\quad$ 0270-6474/05/258104-08\$15.00/0 mechanism, a significant component of synaptic delay would represent the time during which the open receptor channel is initially blocked by an ACh molecule, followed by a slower unblocking after clearance of transmitter (Legendre et al., 2000; Drapeau and Legendre, 2001; Drapeau et al., 2001). The subsequent exit of ACh from the pore would then be reflected in the delayed onset of synaptic current. It follows then that the synaptic delay and onset time of evoked synaptic current at the neuromuscular junction should exhibit a voltage dependence similar to that reported for the channel block. If confirmed, this mechanism would represent a significant departure from the traditional view of neuromuscular transmission. This blocking model has been subsequently proposed for even well characterized neuromuscular synapses such as those in frogs and mammals (Legendre et al., 2000). The ability of ACh to block the ACh receptor channel under experimental conditions is well documented (Sine and Steinbach, 1984; Ogden and Colquhoun, 1985; Liu and Dilger, 1991; Maconochie and Steinbach, 1995), but there has been no direct evidence supporting involvement of ACh block during synaptic transmission (Dionne, 1981). Moreover, the concentration of transmitter required for effective channel block is approximately two orders of magnitude greater than the concentration required to activate $\mathrm{ACh}$ receptors, representing a much higher concentration than thought to actually occur at the neuromuscular junction.

In this study, we probed the synapse between primary motor neurons and fast skeletal muscle for evidence of a voltage dependence of synaptic transmission, taking advantage of the fact that we can control the muscle membrane potential. Our findings show no measurable voltage dependence in either synaptic delay or rise time, arguing strongly against a significant block of the channel by ACh in vivo. 


\section{Materials and Methods}

Zebrafish preparation. All experiments were performed on wild-type zebrafish (Danio rerio), strain Brian's wild type. A decapitated larval fish [96-144 h postfertilization (hpf)] was pinned onto a Sylgard-coated recording chamber, and the skin was peeled from the topside. The fish was treated with $2 \mathrm{~m}$ formamide for $5 \mathrm{~min}$ to prevent muscle contraction, followed by an extensive wash with recording bath solution (see below). All subsequent steps were performed on an Axioskop 2 FS Plus microscope (Zeiss, Oberkochen, Germany) using differential interference contrast (DIC) optics. Under low magnification, the dorsal muscle cells overlaying the spinal cord were carefully removed by means of a glass pipette with a $\sim 25 \mu \mathrm{m}$ opening to which negative pressure was applied (SaintAmant and Drapeau, 2003). On the ventral side, the superficial layer of slow muscle cells was removed to expose deeper fast skeletal muscle cells. The motoneuron electrode and target muscle electrodes were positioned independently using Siskiyou model MX7600 four-axis motorized manipulators and a Siskiyou motorized platform model MXMS, which drove the fixed-stage microscope (Siskiyou Design Instruments, Grants Pass, OR). The manipulators and platform were fixed on adjustable Gantry stands (Sutter Instrument, Novato, CA), allowing the microscope to be moved and focused while maintaining patch clamp of both motor neuron and muscle.

Electrophysiology. The caudal primary $(\mathrm{CaP})$ motor neuron was selected for the paired recordings because of its extensive target field, which includes two-thirds of ventral fast skeletal muscle (Myers et al., 1986; Westerfield et al., 1986). Using DIC optics at $500 \times$ magnification, the $\mathrm{CaP}$ motor neuron could be readily identified on the basis of large size $(\sim 10 \mu \mathrm{m})$ and position near the caudal end of the segmental boundary. To record from the motor neuron, a pipette was fashioned with a long taper to facilitate penetration of the spinal dura. The electrode was typically of $2-3 \mu \mathrm{m}$ outer diameter with a resistance of $12-15 \mathrm{M} \Omega$ when filled with a solution containing the following (in $\mathrm{mM}$ ): $115 \mathrm{~K}$-gluconate, 15 $\mathrm{KCl}, 2 \mathrm{MgCl}_{2}, 10 \mathrm{~K}$-HEPES, 1 BAPTA, $4 \mathrm{Mg}$-ATP, $\mathrm{pH}$ 7.2. All recordings were done at room temperature and in bath solution containing the following (in mM): $134 \mathrm{NaCl}, 2.9 \mathrm{KCl}, 2.1 \mathrm{CaCl}_{2}, 1.2 \mathrm{MgCl}_{2}, 10$ glucose, $10 \mathrm{Na}$-HEPES, pH 7.8. The CaP within the spinal cord was approached with the patch pipette using constant application of positive pressure via a Dale 20 pneumatic transducer (Fluke Electronics, Carson City, NV) to keep the electrode tip clean during penetration of the spinal cord dura. Once a motor neuron was contacted, a gigaohm seal and subsequent whole-cell configuration were obtained using standard techniques. The neuron was held at $-80 \mathrm{mV}$, close to the reported resting membrane potential (Buss et al., 2003).

A paired target fast muscle cell was then selected and whole-cell configuration was obtained with the second recording electrode $(2-3 \mu \mathrm{m}$ outer diameter; 2-4 M $\Omega$ ) containing the following (in $\mathrm{mM}$ ): $120 \mathrm{KCl}, 10$ $\mathrm{K}-\mathrm{HEPES}$, and 5 BAPTA, $\mathrm{pH}$ 7.4. Muscle cells were held at $-50 \mathrm{mV}$ to inactivate sodium channels and minimize muscle contraction. Motor neuron action potentials were elicited by $2 \mathrm{~ms}$ current injections into the soma under current clamp mode. Evoked end-plate currents were recorded in the target muscle cell as it was voltage clamped at different membrane potentials. Whole-cell patch-clamp recordings of spontaneous synaptic currents were performed on fast skeletal muscle cells in 96to 144-hfp-old fish, as described previously (Ono et al., 2001). The pipette and bath solutions were identical with those used for evoked recordings except that $1.5 \mu \mathrm{m}$ tetrodotoxin (TTX) (Alomone Labs, Jerusalem, Israel) was included in the bath solution. The TTX was added to ensure that all synaptic currents were spontaneous and not a result of a nerve action potential. All recordings were performed using either an EPC9/2 or EPC10/2 dual patch-clamp amplifier (List Electronics, Darmstadt-Eberstad, Germany). Data were acquired with Pulse software (HEKA Elektronik, Lambrecht, Germany). The evoked postsynaptic currents were sampled at $50 \mathrm{kHz}$, and the spontaneous synaptic currents were sampled at $100 \mathrm{kHz}$. The series resistance for the muscle cell recordings was $<10 \mathrm{M} \Omega$, and in some pairs, the series resistance was compensated by $50-60 \%$ with a $10 \mu$ s lag time. Recordings before and after series resistance compensation (four pairs) were comparable, so the data were pooled.
Data analysis. Action potentials and synaptic currents were analyzed using Mini-Analysis software (SynaptoSoft, Decatur, GA). The rise time was determined on the basis of $10-90 \%$ of peak amplitude. The delay was computed as the interval between the peak of action potential and EPC onset (defined as $10 \%$ of EPC peak amplitude). Both time points were measured using IGOR Pro software (WaveMetrics, Lake Oswego, OR) with NeuroMatic extension (Laboratory of Synaptic Transmission and Information Processing, University College London, London, UK). For miniature EPC (mEPC) analysis, events with $10-90 \%$ rise times longer than $0.4 \mathrm{~ms}$ and under $50 \mathrm{pA}$ in amplitude were excluded, because these slow and small events are electrotonic reflections of currents generated in neighboring muscle cells (Nguyen et al., 1999; Buss and Drapeau, 2000; Luna et al., 2004). All data represent mean \pm SD unless otherwise specified, and statistical significance was assessed by a two-tailed $t$ test.

\section{Results}

\section{Determination of quantal features}

The CaP motor neuron is one of three primary motor neurons present in each tail segment and can be identified in the spinal cord on the basis of its stereotypic position (Myers et al., 1986; Westerfield et al., 1986). Because the CaP neuron innervates approximately two-thirds of the ventral fast skeletal muscle fibers

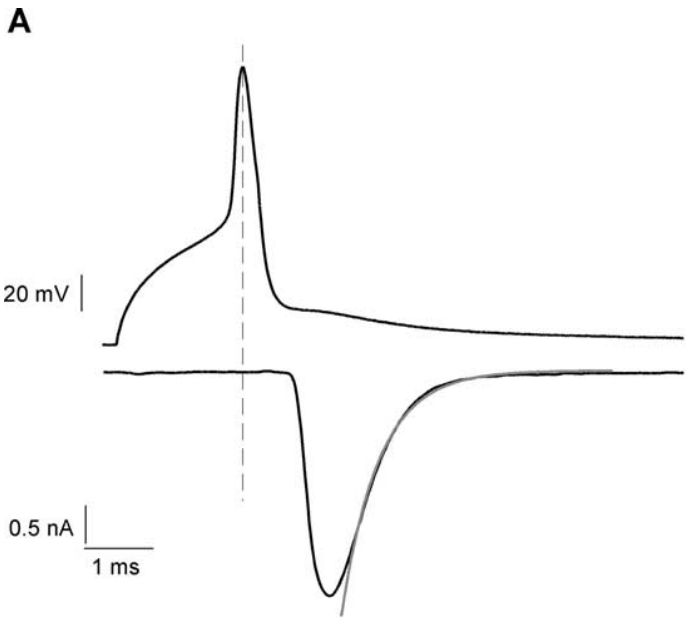

B
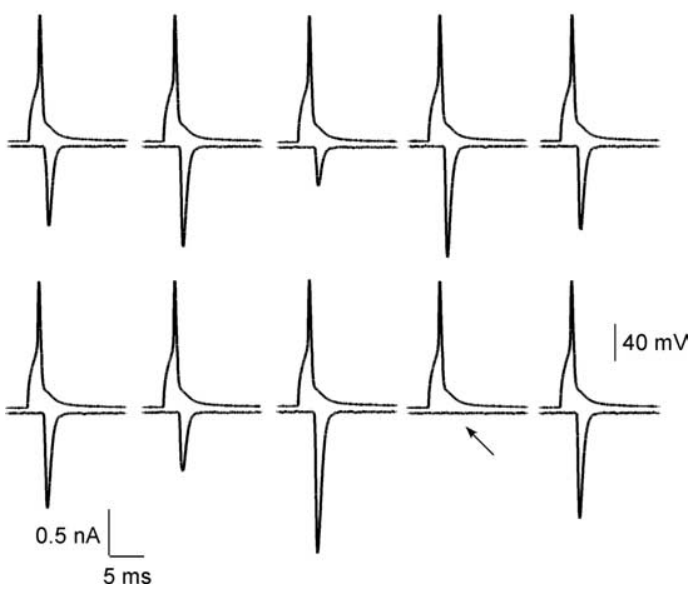

Figure 1. Paired motor neuron-muscle recordings. $A$, An action potential from a CaP motor neuron (top) and evoked synaptic current from target fast muscle (bottom). The EPC is fit with a single exponential ( $\tau=0.56 \mathrm{~ms}$ ). $\boldsymbol{B}$, Responses to $1 \mathrm{~Hz}$ stimulation showing an invariant action potential and associated EPC exhibiting large fluctuations in amplitude (a failure is indicated by an arrow). Muscle was held at $-50 \mathrm{mV}$, and the CaP membrane potential was $-80 \mathrm{mV}$. 
A

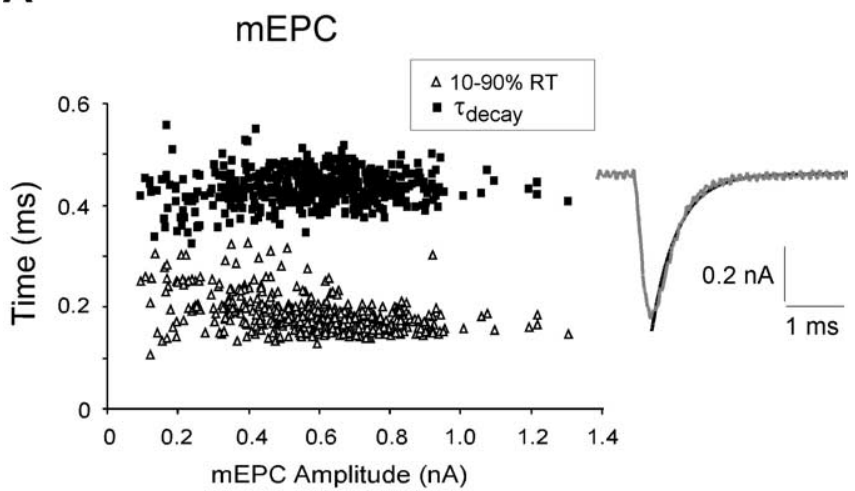

B

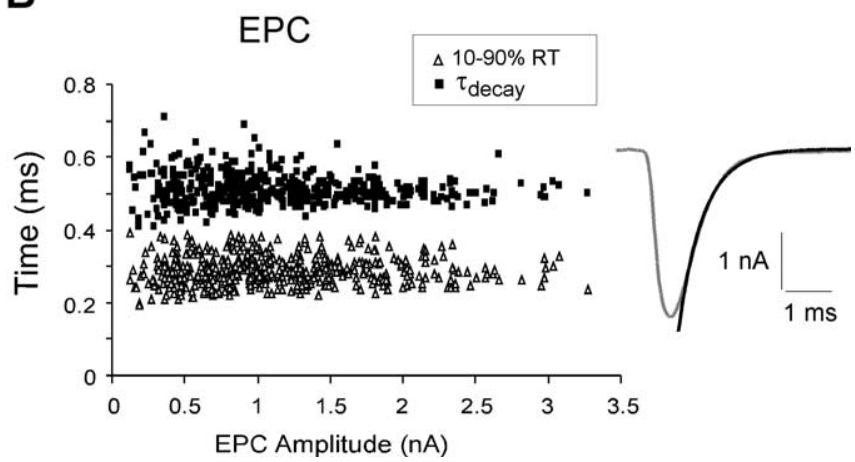

Figure 2. Kinetics of spontaneous and evoked synaptic currents. Scatter plots of rise time (RT) (triangles) and decay time constant (squares) versus amplitude for spontaneous $(\boldsymbol{A})$ and evoked (B) synaptic currents. In total, $460 \mathrm{mEPCs}$ from a single muscle cell and 470 EPCs from a single paired recording were used to generate distributions. A representative $\mathrm{mEPC}$ and EPC are shown to the right wherein the decay phases were fitted with a single exponential curve. The time constants measured 0.43 and $0.56 \mathrm{~ms}$, respectively. Muscle cells were held at $-50 \mathrm{mV}$.

within an individual segment, a target muscle cell was usually obtained on the first attempt at paired recording. However, in those cases in which the muscle tested was not innervated by the $\mathrm{CaP}$ neuron, it was still possible to hold the neuron long enough to try additional muscle cells. Under current clamp, the input resistance of the $\mathrm{CaP}$ neuron averaged $123 \pm 25 \mathrm{M} \Omega(n=24$ cells). The membrane potential of the motor neuron was adjusted to $-80 \mathrm{mV}$, close to the resting membrane potential (Buss et al., 2003). To elicit an action potential, a $2 \mathrm{~ms}$ pulse of outward current between 400 and 550 pA was usually sufficient. The resultant spikes were robust and invariant in waveform for frequencies up to $100 \mathrm{~Hz}$. The motor neuron action potentials were characterized by duration of $0.52 \pm 0.15 \mathrm{~ms}$ at half-amplitude (average amplitude, $125 \pm 13 \mathrm{mV} ; n=24$ cells) and a small afterdepolarization lasting several milliseconds (Fig. 1).

Table 1. Properties of wild-type EPC at different membrane potentials

\begin{tabular}{lccll}
\hline $\begin{array}{l}\text { Membrane } \\
\text { potential } \\
(\mathrm{mV})\end{array}$ & $\begin{array}{l}\text { Normalized } \\
\text { amplitude }\end{array}$ & $\begin{array}{l}\text { Normalized } \\
\text { delay }\end{array}$ & $\begin{array}{l}10-90 \% \\
\text { Rise time } \\
(\mathrm{ms})\end{array}$ & $\begin{array}{l}\text { Decay } \tau \\
(\mathrm{ms})\end{array}$ \\
\hline-50 & $-1 \pm 0$ & $1 \pm 0$ & $0.30 \pm 0.05$ & $0.64 \pm 0.07$ \\
-30 & $-0.63 \pm 0.08$ & $0.99 \pm 0.03$ & $0.28 \pm 0.03$ & $0.61 \pm 0.06$ \\
-10 & $-0.28 \pm 0.07$ & $0.99 \pm 0.04$ & $0.29 \pm 0.05$ & $0.57 \pm 0.08$ \\
+20 & $0.26 \pm 0.17$ & $0.99 \pm 0.02$ & $0.25 \pm 0.02$ & $0.47 \pm 0.09$ \\
+40 & $0.54 \pm 0.24$ & $1.00 \pm 0.02$ & $0.26 \pm 0.03$ & $0.47 \pm 0.07$ \\
\hline
\end{tabular}

Data were from 12 paired recordings. EPC amplitude and synaptic delay are normalized to the values at $-50 \mathrm{mV}$.
A

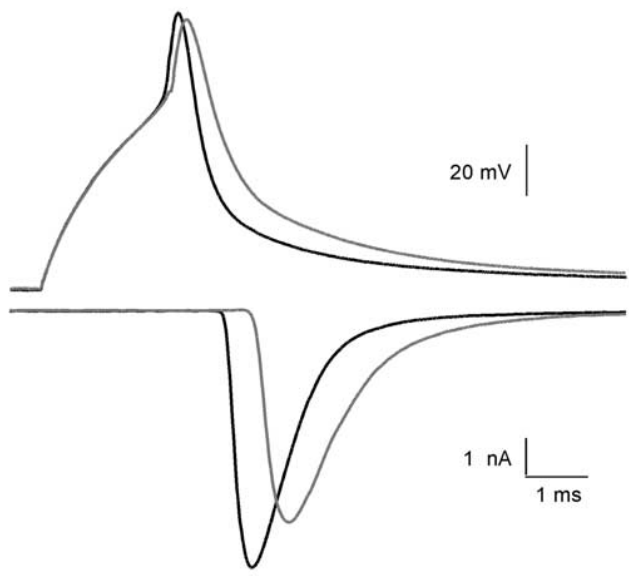

B

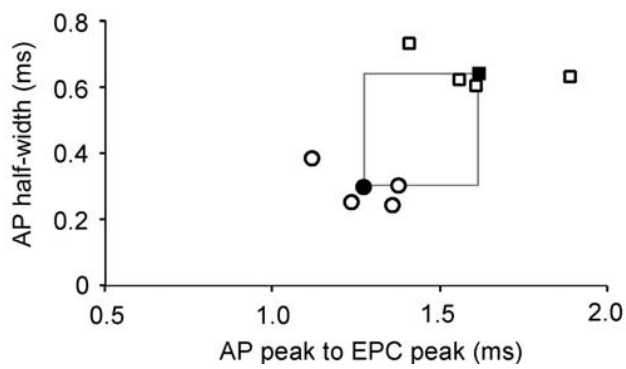

Figure 3. Delay and rise time are increased in response to prolongation of the action potential. $\boldsymbol{A}$, Sample traces of motor neuron action potential and postsynaptic response shown before (black) and after (gray) bath perfusion of $0.2 \mu \mathrm{m}$ 4-AP. B, The relationship between the increase in action potential duration and time-to-peak EPC. Pretreatment with 4-AP (circles) and post4-AP (squares) are indicated. The duration of half-repolarization is plotted on the ordinate, and the time from action potential peak to peak of the EPC is plotted on the abscissa. The rectangle shows the distance between mean (filled symbols) horizontal and vertical coordinates.

The target fast muscle cells in the ventral mid-region of 96144 hpf fish were reasonably electrically compact despite the presence of weak electrical coupling (Nguyen et al., 1999; Luna et al., 2004). Because of the small size of muscle, series resistance compensation resulted in no significant change in synaptic current waveform. In paired recordings, a neuronal action potential faithfully elicited an evoked synaptic current (EPC) in 15 of 20 muscle cells tested (Fig. 1). In the other 5 cells, synaptic failures occurred (Fig. 1B). The mean EPC amplitude versus membrane potential placed the reversal potential at approximately $+7 \pm 5$ $\mathrm{mV}$ (see Fig. $4 A, B$ ). In every pair tested, the EPCs showed considerable fluctuations in amplitude (Fig. $1 B$ ). Because of a very large size (mean, $444 \pm 254 \mathrm{pA}$ at $-50 \mathrm{mV}$ ), nearly all of the spontaneous events generated directly in the fast muscle cell were resolved. The large unitary size of events underlies the large stepwise fluctuations in EPC amplitude that was seen in vivo. These fluctuations, along with the observed failures in 5 of 20 pairs tested, further reflected the large quantal size and pointed to a small quantal content at these synapses in vivo.

Kinetics of in vivo synaptic responses The evoked EPCs and spontaneous mEPCs of larval zebrafish fast muscle both exhibited remarkably fast kinetics. The decay phases of both mEPCs and EPCs in fast muscle were generally well fit by a single 
A

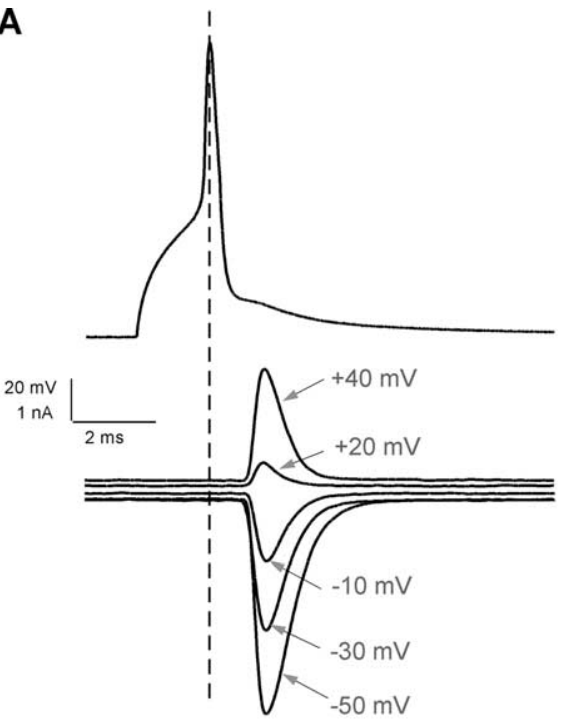

B

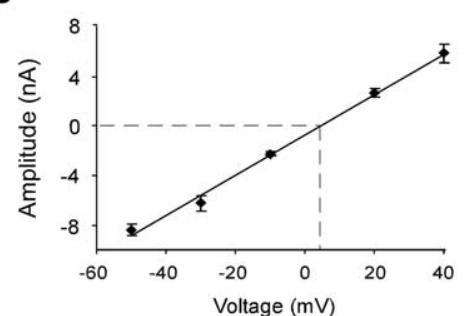

C

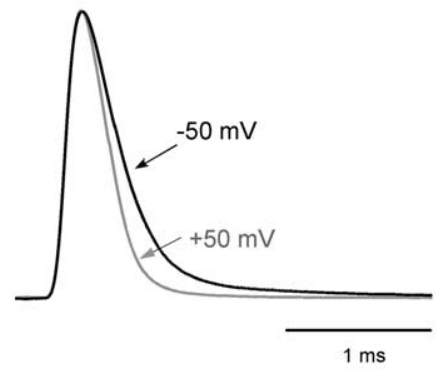

D

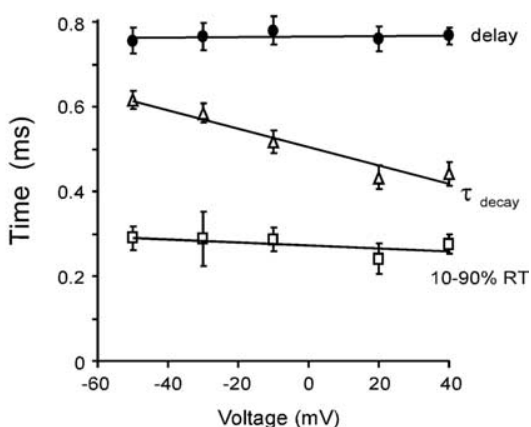

Figure 4. Voltage dependence of synaptic transmission. $\boldsymbol{A}$, Sample traces of a CaP action potential (top trace) and associated EPCS (bottom traces) in muscle held at five membrane potentials between -50 and $+40 \mathrm{mV}$. $\boldsymbol{B}$, Relationships between mean EPC amplitude and muscle membrane potential. The membrane potential corresponding to 0 -current level is shown by the dotted line $(+5.6 \mathrm{mV})$. C, Comparison of the EPC time course at -50 and $+50 \mathrm{mV}$ (shown at same polarity for comparison). Each trace represents an average of 10 normalized EPCs. The decay phases fitted by single exponential curves measured 0.54 and $0.35 \mathrm{~ms}$ for -50 and $+50 \mathrm{mV}$, respectively. $\boldsymbol{D}$, The dependence of delay (circle), decay time constant (triangle), and $10-90 \%$ rise time (square) on the muscle holding potential. Data are derived from a single paired recording, and each point represents the mean \pm SD of 10 -30 EPCS. The graphed points were fitted with lines that were generated using the least-squares method. RT, Rise time.

exponential (Figs. 1, 2). At $-50 \mathrm{mV}$, the mean time constant for EPC decay measured $0.64 \pm 0.07 \mathrm{~ms}(n=12$ pairs), which was somewhat slower than the $0.45 \pm 0.11 \mathrm{~ms}$ measured for mEPC decay (Fig. 2, Table 1). The $10-90 \%$ rise time of the EPC measured $0.30 \pm 0.05 \mathrm{~ms}$, which was also slightly longer than the $0.22 \pm 0.06 \mathrm{~ms}$ obtained for $\mathrm{mEPC}$ rise time (Fig. 2, Table 1). Because the rise times were very fast, we likely overestimated the actual rise times because of the frequency constraints of the recording methodology. Both the slower rise and decay exhibited for EPCs compared with mEPCs were consistent with the blocking model, which predicts that larger amplitude events would provide greater slowing because of a higher cleft concentration of ACh. Alternatively, the difference between EPCs and mEPC kinetics reflected a lack of synchrony associated with release of multiple quanta (Dempster, 1986; Van der Kloot, 1988). This idea was supported by the observation that $\mathrm{MEPC}$ and EPC kinetics were both independent of amplitude (Fig. $2 A, B$ ). Furthermore, cumulative scatter plots of amplitude versus either rise time or decay time constant indicated no dependence on event amplitude that spans two orders of magnitude (data not shown). Assuming that the differences in amplitude of events reflect, to some extent, differences in cleft ACh concentration, it appears that the kinetics is independent of the amount of ACh released in vivo.

Using paired recordings, it was possible to measure the delay between the action potential and the onset of evoked synaptic current (Fig. 1A). A block by ACh should lead to a prolonged delay, corresponding to the time required for clearance of cleft ACh. This delay, measured from the peak of somatic action potential to $10 \%$ of the peak EPC amplitude, was $0.78 \pm 0.10 \mathrm{~ms}$ (20 pairs). This overall delay is in general agreement with the $0.75 \mathrm{~ms}$ synaptic delay measured at frog neuromuscular junction (Katz and Miledi, 1965), suggesting that synaptic delay is the principal contributor to delay rather than propagation time or block time. Studies have also shown that the synaptic delay reflects time between initiation of the presynaptic action potential and the onset of significant repolarization (Katz and Miledi, 1967). This occurs because transmitter is released during the repolarizing phase of the action potential, when maximal $\mathrm{Ca}^{2+}$ channel activation has occurred and the driving force for entering calcium is suddenly increased (Katz and Miledi, 1967). To test the correspondence between repolarization and delay at the neuromuscular junction, repolarization was purposely slowed through application of K channel blockers (Fig. 3). Blockers of $\mathrm{Ca}^{2+}$-activated $\mathrm{K}$ current were first tested because they have been shown to regulate transmitter release at amphibian neuromuscular junctions (Yazejian et al., 1997; Pattillo et al., 2001). However, neither $100 \mathrm{~nm}$ charybdotoxin, $100 \mathrm{~nm}$ iberiotoxin, nor $10 \mu \mathrm{M}$ paxilline affected the somatic action potential or synaptic current in six trials. In contrast, treatment with 4-aminopyridine (4-AP) (0.1-1 mM) prolonged somatic action potential waveforms (Fig. 3). Over time, 4-aminopyridine resulted in the firing of repetitive spikes in response to a single stimulus, restricting our analysis to the initial actions of this drug. The half-duration of action potential repolarization (time from peak to half-amplitude of repolarization) was increased by $0.37 \pm$ $0.11 \mathrm{~ms}$ in response to 4 -AP (Fig. 3B). The corresponding lengthening of time between the peak of the action potential and peak of the EPC measured $0.34 \pm 0.17 \mathrm{~ms}(n=4$ pairs) (Fig. $3 B)$. This increase was attributable, collectively, to lengthened onset (by $0.20 \pm 0.09 \mathrm{~ms}$ ) and $10-90 \%$ rise time (by $0.06 \pm 0.03 \mathrm{~ms}$ ) of synaptic current. Additionally, an increased decay time constant (by $0.29 \pm 0.27 \mathrm{~ms} ; n=4$ pairs) was observed in response to 4 -AP. The slowed rise and decay time of the EPC after treatment with 4-AP probably reflect a compromised synchrony of transmitter release when the action potential was broadened. These data indicate that the major determinant of the delay is likely to be encoded in the presynaptic action potential waveform. The effects of 4-AP further demonstrated our ability to detect changes in synaptic delay.

\section{Voltage dependence of EPCs argues against a physiological block by ACh}

The putative block of ACh receptor channels by the positively charged ACh is voltage dependent, becoming stronger with more negative potentials and disappearing with positive membrane 
potentials (Sine and Steinbach, 1984; Ogden and Colquhoun, 1985; Sine et al., 1990). Therefore, as a test of open channel block of ACh receptor channels by ACh, we examined the synaptic currents over a range of muscle membrane potentials (Fig. 4, Table 1). A transient block of the channel, as proposed, would be reflected in a reduced amplitude, delayed EPC onset, and slowed kinetics at negative potentials compared with positive potentials.

The average peak EPC amplitudes exhibited a linear dependence on muscle membrane potentials between -50 and $+40 \mathrm{mV}$ (Fig. $4 \mathrm{~B}$ ). Comparison of delay measurements over this range of muscle membrane potentials revealed no evidence of prolongation at negative membrane potentials that might signal a block by ACh (Figs. $4 D$, 7B; Table 1). Should the block at negative potentials be incomplete, it might be reflected in a slowed rise time of synaptic current at negative potentials. Comparisons of average waveforms obtained at $-50 \mathrm{mV}$ to those at $+50 \mathrm{mV}$ showed no obvious difference in rise times (Figs. 4C,D, 7A; Table 1). When the overall values were computed, a slightly faster rise time was seen at positive potentials ( 40 $\mu$ s on average) than negative potentials (Fig. $4 D$, Table 1). This rise time was unchanged after inhibition of esterase by treatment with fasciculin II (Fas II) (see Figs. $6 D, 7 A$ ). The small differences may result from incomplete series resistance compensation at very positive potentials. Alternatively, the difference in rise times at -50 and $+40 \mathrm{mV}$ may reflect the very weak voltage dependence of the EPC rise time that was identified by Magelby and Stevens (1972b). The time constant of decay indicated a weak voltage dependence decreasing from $0.64 \pm 0.07$ to $0.47 \pm$ $0.07 \mathrm{~ms}$ between -50 and $+40 \mathrm{mV}$ (Figs. $4 C, D, 7 C$; Table 1). This voltage dependence likely reflects the intrinsic voltage dependence of the channel closing rate that has been described for all species of muscle nicotinic receptors. This voltage dependence is independent of ACh concentration and does not reflect a channel block (Magleby and Stevens, 1972a; Anderson and Stevens, 1973; Steinbach, 1989).

\section{Synaptic current kinetics after} inhibition of acetylcholinesterase also shows no sign of ACh block

To further test whether ACh-mediated channel block of postsynaptic receptors could potentially play a physiological role in synaptic transmission, we inhibited acetylcholinesterase (AChE) to increase ACh concentration in the synaptic cleft. AChE was inactivated by treatment with Fas II, a pep-

B

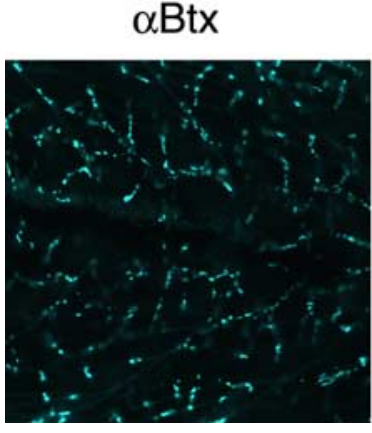

Fas II

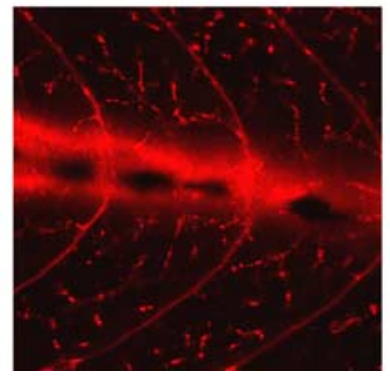

Merge

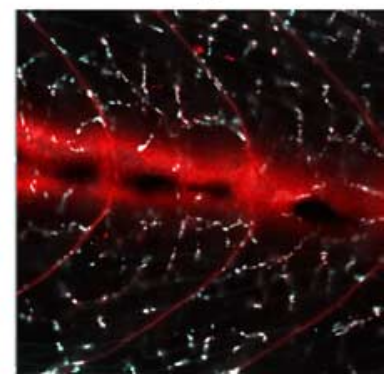

Figure 5. Confocal images of acetylcholine receptor and acetylcholinesterase distribution in $96-120 \mathrm{hpf}$ zebrafish tail muscle. Acetylcholine receptors are labeled by $20 \mathrm{~min}$ treatment with tetramethylrhodamine $\alpha$-bungarotoxin $(\alpha \mathrm{Btx})$, and acetylcholinesterase is labeled by 45 min treatment with Alexa Fluor 647-conjugated Fas II [methods detailed in the studies by lbañezTallon et al. (2004) and Ono et al. (2001)]. Merged image indicates the colocalization of the receptor and acetylcholinesterase.
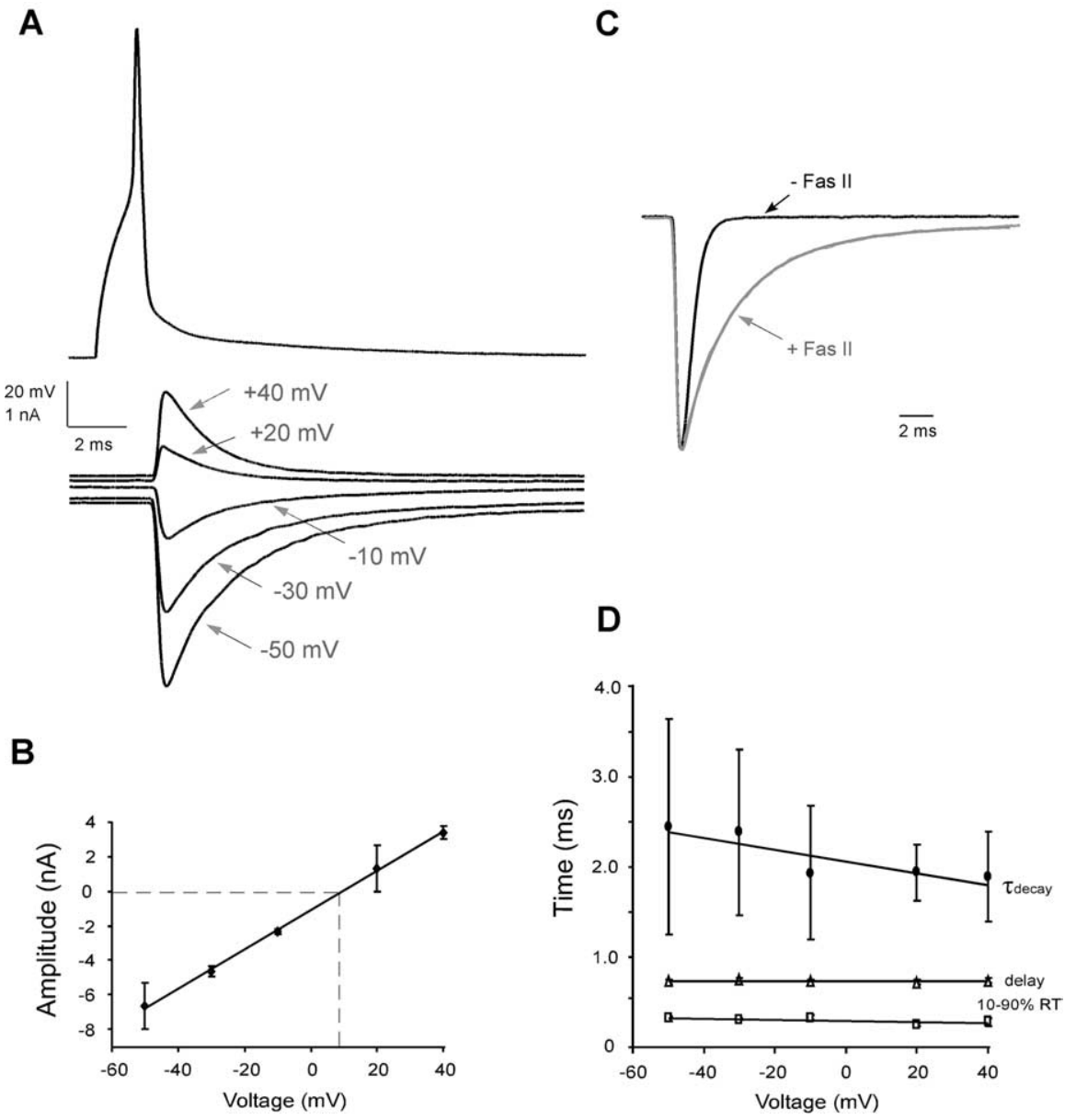

Figure 6. Paired recording after inhibition of acetylcholinesterase by Fas II. $\boldsymbol{A}$, The motor neuron action potential (top) and muscle EPC (bottom) traces after a 20 min incubation in $300 \mathrm{~nm}$ Fas II. Representative EPCs are shown for each of five different muscle membrane potentials. $\boldsymbol{B}$, Relationships between mean EPC amplitude and muscle membrane potential. The membrane potential corresponding to 0 -current level is shown by the dotted line $(+9.7 \mathrm{mV})$. C, Normalized EPCs before and after (shaded) treatment with Fas II. Each trace represents the average of $10-30$ events from a single recording at $-50 \mathrm{mV}$. D, Dependence of delay (circles), decay time constant (triangles), and $10-90 \%$ rise time (squares) on muscle membrane potential. Each point represents the mean \pm SD of 10-30 events from a single recording. The graphed points were fitted with lines that were generated using the least-squares method. RT, Rise time. tide inhibitor highly selective for peripheral forms of the esterase (Karlsson et al., 1984). This toxin has been shown to bind tightly to AChE of zebrafish (Ibañez-Tallon et al., 2004). Colabeling of muscle with Alexa 647 Fas II and rhodamine $\alpha$-bungarotoxin 
A
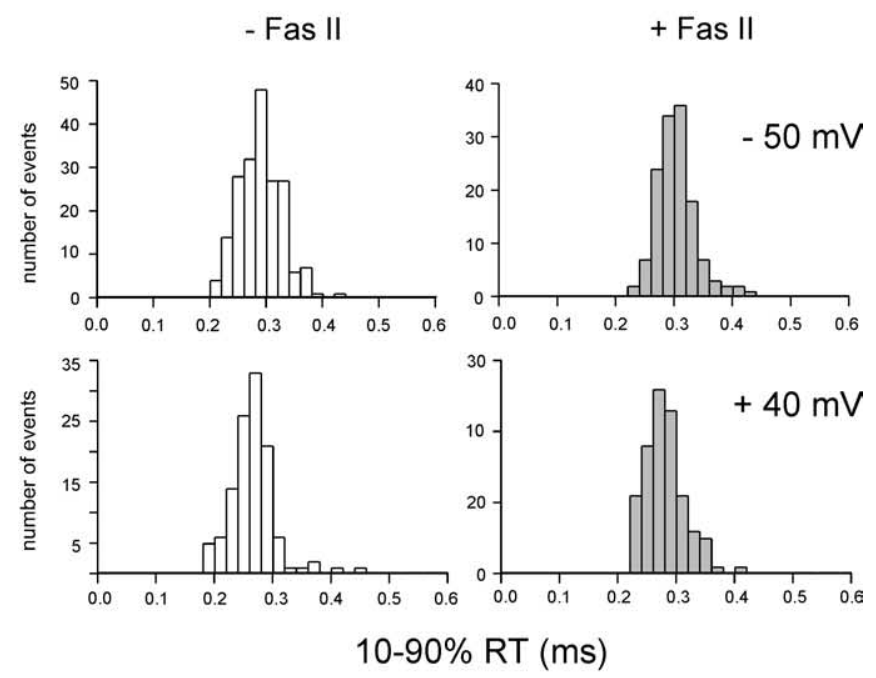

B
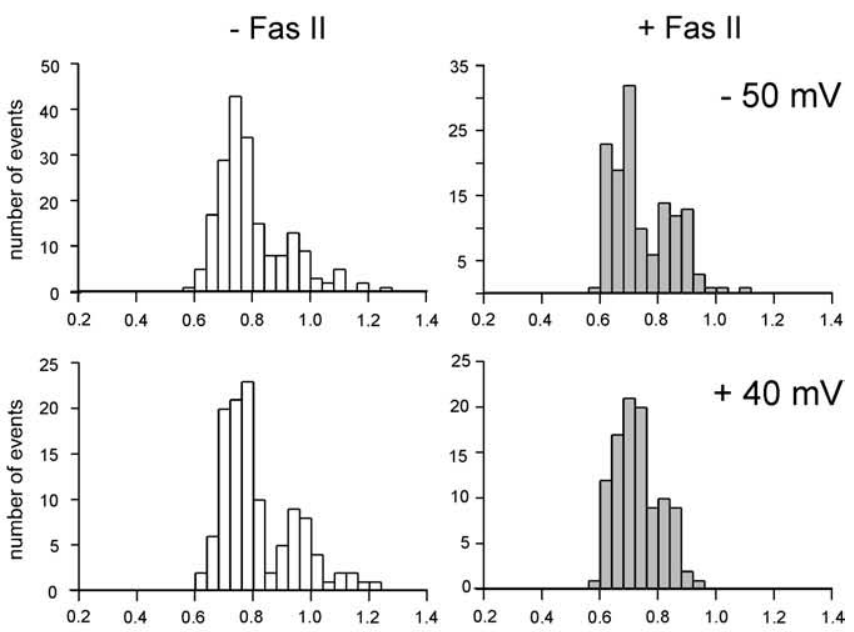

delay (ms)

C
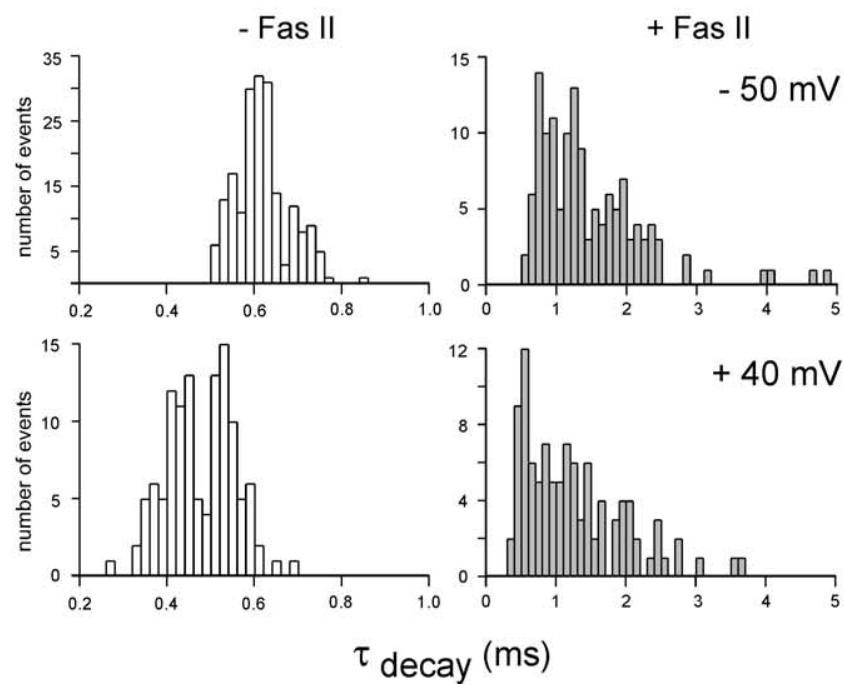

indicates that the cholinesterase is localized to synapses (Fig. 5). As would be expected from repetitive binding of ACh to postsynaptic receptor, the time course of EPC decay is markedly prolonged after treatment with 250-300 nM Fas II (Figs. 6, 7C). To approximate the magnitude of prolongation, the time constants of decay were compared for control and Fas II-treated muscle. In some cases, the decay phase of Fas II-treated muscle displayed a biexponential time course. For such EPCs, the fast component of the decay was compared with control EPC decay. Overall, Fas II treatment prolonged decay time constant by twofold to threefold, from $0.64 \pm 0.07(n=12$ pairs $)$ to $1.67 \pm 0.70 \mathrm{~ms}(n=9$ pairs $)$ at $-50 \mathrm{mV}$ (Figs. $6 A, C ; 7 C$ ). The prolongation of decay was not associated with an increased rise time, which averaged $0.30 \pm$ $0.02 \mathrm{~ms}(n=9$ pairs $)$, compared with $0.30 \pm 0.05 \mathrm{~ms}(n=12$ pairs) measured for nontreated cells at $-50 \mathrm{mV}$ (Figs. 6, 7A). Likewise, the delay in Fas II-treated muscle cells $(0.71 \pm 0.09 \mathrm{~ms}$; $n=9$ ) was not significantly different from nontreated muscle $(p=0.03)$ (Figs. 6, 7B). The average amplitude of EPCs was increased by $54 \%$, which is consistent with an increase in peak ACh concentration after inhibition of cholinesterase (Chang et al., 1985; Minic et al., 2002).

As with control muscle, there was no trend for either rise time (Fig. 6D, Table 2) or delay (Fig. 6D, Table 2) to exhibit a voltage dependence after inhibition of esterase. Also, neither of these kinetic parameters was affected by treatment with Fas II (Fig. 7; Tables 1, 2). In contrast, the decay distributions were greatly prolonged after Fas II treatment and retained the mild voltage dependence characteristic of control muscle synaptic current (Fig. 7; Tables 1, 2). Finally, as seen with nontreated cells, the mean EPC amplitude showed a linear dependence on the muscle membrane potential (Fig. $6 A, B$ ).

\section{Discussion}

The traditional view of chemical synaptic transmission holds that the onset kinetics of synaptic current is determined through the binding of ligand to active sites on the receptor and the resultant opening of the channel. Once activated, the current usually follows an exponential decay whose time constant is governed by the intrinsic closing rate of the receptor. However, according to a recently proposed rebound model, the onset and decay of synaptic current at the neuromuscular junction would be determined by the clearance time of ACh from the cleft and the subsequent discharge of molecule from the open, but blocked pore (Legendre et al., 2000; Drapeau and Legendre, 2001; Drapeau et al., 2001). Moreover, the authors further propose that the blocking model also applies to other well studied nerve muscle preparations such as those of frog and mammals (Legendre et al., 2000; Drapeau and Legendre, 2001). Historically, the idea that ACh provides a physiological block at synapses has been dismissed principally on the grounds that millimolar concentrations are needed to block the open channel (Sine and Steinbach, 1984; Ogden and Colquhoun, 1985; Liu and Dilger, 1991; Maconochie and Steinbach, 1995). For example, the dissociation constant for ACh block in zebrafish is $\sim 0.5 \mathrm{~mm}$ (Drapeau and Legendre, 2001), a concentration well above saturation for binding to receptor and not likely achieved at neuromuscular synapses (Naranjo and Brehm, 1993; Paradiso and Brehm, 1998).

Figure 7. Comparisons of EPC kinetics in control (left) and acetylcholinesterase inhibited (right) muscle at $-50 \mathrm{mV}$ (top) and $+40 \mathrm{mV}$ (bottom) muscle membrane potential. The $10-90 \%$ rise time $(\boldsymbol{A})$, delay $(\boldsymbol{B})$, and decay time constant $(\boldsymbol{C})$ were computed for nine different pairs treated with 250-300 nм Fas II and 12 nontreated control pairs. RT, Rise time. 
The rebound proposal was based on apparent waveform differences between spontaneous synaptic currents recorded in vivo and macroscopic kinetics of AChinduced currents in response to fast perfusion of outside-out patches (Legendre et al., 2000). In general, the inward current seen on termination of ACh application decayed much faster than the average decay time constant measured for spontaneous synaptic currents. Only when the concentration of applied ACh was elevated to $10 \mathrm{~mm}$, a concentration shown to provide effective block of ACh receptors, did the current decay provide good agreement with the time course of synaptic current decay. Importantly, this prolonged decay after removal of ACh was seen only at negative potentials at which a block would be most effective. The proposed functional importance of this prolongation of synaptic current would be to promote charge spread in the muscle fiber (Legendre et al., 2000; Drapeau and Legendre, 2001).

Our recordings of evoked synaptic current kinetics measured over the potential range published for fast perfusion experiments do not support ACh receptor block as playing a significant role. In outside-out patches of zebrafish muscle, the block by ACh was very sensitive to membrane potential, exhibiting an $e$-fold increase per $30 \mathrm{mV}$ increase in negativity (Legendre et al., 2000; Drapeau and Legendre, 2001). Correspondingly, we would expect the delay, amplitude, rise time, and/or decay of synaptic current to reflect this steep dependence on membrane potential. However, our overall findings indicate that these parameters of synaptic currents are not dependent on membrane potential in a way that would point to a block by ACh. First, the EPC delay is completely insensitive to membrane potentials between -50 and $+40 \mathrm{mV}$, even after block of acetylcholinesterase. Second, the block model predicts a $25 \%$ reduction in amplitude at negative potentials (Legendre et al., 2000). However, we find a linear relationship between amplitude and membrane potential over the range tested, even after block of acetylcholinesterase. Third, the model predicts significant slowing of both rise and decay times because of the very slow unblocking times estimated by fast perfusion (Legendre et al., 2000). The slowing of rise time based on fast perfusion measured $\sim 40 \mu \mathrm{s}$, similar to our EPC measurements. However, the $40 \mu$ s difference is maintained after inhibition of acetylcholinesterase, despite the twofold to threefold slowing of decay kinetics. Should the difference at positive and negative potentials be the result of a block by $\mathrm{ACh}$, as originally proposed, then increasing and prolonging cleft ACh concentration should have further slowed the rise time. Therefore, this $10-15 \%$ reduction in rise time at positive potentials likely reflects the very weak voltage dependence originally described for EPC rise time at frog nerve-muscle synapses (Magleby and Stevens, 1972). The decay of synaptic current also exhibits a modest voltage sensitivity, but it is 10-20 times less than predicted for the voltage-dependent ACh block. In fact, the voltage dependence measured is even weaker than the intrinsic voltage dependence of closing rate shown for frog and mammalian muscle nicotinic receptors (Steinbach, 1989). Therefore, it is highly likely that voltage dependence of current decay is a reflection of the intrinsic voltage dependence of the channel rather than of the block. Finally, additional evidence against a block model is provided by scatter plots of event amplitude versus both rise time and decay time. Neither mEPCs nor EPCs show a trend for larger amplitude currents to exhibit slower kinetics. Presumably larger amplitude events reflect greater ACh concentration. Thus, large amplitude synaptic currents should be prolonged compared with smaller amplitude currents.

The delay was completely independent of membrane potential, demonstrating that a block governs no component of onset. If not a block by ACh, what accounts for the 600-1000 $\mu$ s delay? The major component is likely synaptic delay. This conclusion is based on the agreement between our measured delay and the synaptic delay measured directly (Katz and Miledi, 1965). Furthermore, as first shown for frog neuromuscular junction by Katz and Miledi (1967), the predominant component of synaptic delay is the time interval between initiation of the presynaptic action potential and entry into the repolarization phase. In other words, transmitter release is associated with repolarization wherein presynaptic calcium entry occurs through an increase in driving force. Consistent with this idea, we observed an increase in synaptic delay when the motor neuron action potential in zebrafish was prolonged by block of outward K current. The $\mathrm{K}$ channel type that governs repolarization in zebrafish is not the calcium-activated $\mathrm{K}$ channel as shown for Xenopus spinal neurons (Yazejian et al., 1997; Pattillo et al., 2001). Identification of the $\mathrm{K}$ channel will be important, because this channel type is a candidate for synaptic modulation.

Our paired recordings represent the first in vivo patch-clamp recordings of evoked end-plate currents in any vertebrate preparation. The evoked synaptic currents showed remarkable synaptic speed as reflected in the decay time constants that averaged $0.64 \mathrm{~ms}$ at $-50 \mathrm{mV}$. This speed of synaptic current was indicated in measurements of synaptic potentials (Buss and Drapeau, 2000) and in the decay of spontaneous synaptic currents (Nguyen et al., 1999; Luna et al., 2004). Now the decay times obtained for EPCs confirm the synaptic speed. Also, instead of a quantal content between 100 and 300, as determined for frog skeletal muscle (Katz and Miledi, 1979), the mean quantal content at the zebrafish nerve muscle synapse was $<10$. This low quantal content appeared to hold for ages between 3 and $14 \mathrm{~d}$, suggesting that this is not a reflection of incomplete synapse development. The physical basis for so few quanta being released in zebrafish is not clear. However, labeling of synaptic vesicles by either SV2 antibody or FM1-43 indicated that substantial numbers of vesicles were present (data not shown). An alternative potential mechanism causal to this small quantal content is a greatly reduced probability of vesicle release in zebrafish tail muscle compared with limb muscle of other vertebrates. The physiological basis of reduced release might lie in the high input resistance of these compact muscle cells and/or the presence of polyneuronal innervation in individual cells. Thus, the level of presynaptic drive required to depolarize vertebrate skeletal muscle may be adjusted according to the postsynaptic properties. Paired nerve-muscle recordings from zebrafish now offer a powerful means to test for the activity- 
dependent feedback on presynaptic function and synaptic modulation.

\section{References}

Anderson CR, Stevens CF (1973) Voltage clamp analysis of acetylcholine produced end-plate current fluctuations at frog neuromuscular junction. J Physiol (Lond) 235:655-691.

Buss RR, Drapeau P (2000) Physiological properties of zebrafish embryonic red and white muscle fibers during early development. J Neurophysiol 84:1545-1557.

Buss RR, Bourque CW, Drapeau P (2003) Membrane properties related to the firing behavior of zebrafish motoneurons. J Neurophysiol 89:657-664.

Chang CC, Hong SJ, Lin HL, Su MJ (1985) Acetylcholine hydrolysis during neuromuscular transmission in the synaptic cleft of skeletal muscle of mouse and chick. Neuropharmacology 24:533-539.

Dempster J (1986) The use of the driving function in the analysis of endplate current kinetics. J Neurosci Methods 18:277-285.

Dionne VE (1981) The kinetics of slow muscle acetylcholine-operated channels in the garter snake. J Physiol (Lond) 310:159-190.

Drapeau P, Legendre P (2001) Neuromuscular transmission on the rebound. Receptors Channels 7:491-496.

Drapeau P, Buss RR, Ali DW, Legendre P, Rotundo RL (2001) Limits to the development of fast neuromuscular transmission in zebrafish. J Neurophysiol 86:2951-2956.

Ibañez-Tallon I, Wen H, Miwa JM, Xing J, Tekinay AB, Ono F, Brehm P, Heintz N (2004) Tethering naturally occurring peptide toxins for cellautonomous modulation of ion channels and receptors in vivo. Neuron 43:305-311.

Karlsson E, Mbugua PM, Rodriguez-Ithurralde D (1984) Fasciculins, anticholinesterase toxins from the venom of the green mamba Dendroaspis angusticeps. J Physiol (Paris) 79:232-240.

Katz B, Miledi R (1965) The measurement of synaptic delay, and the time course of acetylcholine release at the neuromuscular junction. Proc R Soc Lond B Biol Sci 161:483-495.

Katz B, Miledi R (1967) The release of acetylcholine from nerve endings by graded electric pulses. Proc R Soc Lond B Biol Sci 167:23-38.

Katz B, Miledi R (1979) Estimates of quantal content during "chemical potentiation" of transmitter release. Proc $\mathrm{R}$ Soc Lond B Biol Sci 205:369-378.

Legendre P, Ali DW, Drapeau P (2000) Recovery from open channel block by acetylcholine during neuromuscular transmission in zebrafish. J Neurosci 20:140-148.

Liu Y, Dilger JP (1991) Opening rate of acetylcholine receptor channels. Biophys J 60:424-432.

Luna VM, Wang M, Ono F, Gleason MR, Dallman JE, Mandel G, Brehm P (2004) Persistent electrical coupling and locomotory dysfunction in the zebrafish mutant shocked. J Neurophysiol 92:2003-2009.

Maconochie DJ, Steinbach JH (1995) Block by acetylcholine of mouse mus- cle nicotinic receptors, stably expressed in fibroblasts. J Gen Physiol 106:113-147.

Magleby KL, Stevens CF (1972a) The effect of voltage on the time course of end-plate currents. J Physiol (Lond) 223:151-171.

Magleby KL, Stevens CF (1972b) A quantitative description of end-plate currents. J Physiol (Lond) 223:173-197.

Minic J, Molgo J, Karlsson E, Krejci E (2002) Regulation of acetylcholine release by muscarinic receptors at the mouse neuromuscular junction depends on the activity of acetylcholinesterase. Eur J Neurosci 15:439-448.

Myers PZ, Eisen JS, Westerfield M (1986) Development and axonal outgrowth of identified motoneurons in the zebrafish. J Neurosci 6:2278-2289.

Naranjo D, Brehm P (1993) Modal shifts in acetylcholine receptor channel gating confer subunit-dependent desensitization. Science 260:1811-1814.

Nguyen PV, Aniksztejn L, Catarsi S, Drapeau P (1999) Maturation of neuromuscular transmission during early development in zebrafish. J Neurophysiol 81:2852-2861.

Ogden DC, Colquhoun D (1985) Ion channel block by acetylcholine, carbachol and suberyldicholine at the frog neuromuscular junction. Proc R Soc Lond B Biol Sci 225:329-355.

Ono F, Higashijima S, Shcherbatko A, Fetcho JR, Brehm P (2001) Paralytic zebrafish lacking acetylcholine receptors fail to localize rapsyn clusters to the synapse. J Neurosci 21:5439-5448.

Paradiso K, Brehm P (1998) Long-term desensitization of nicotinic acetylcholine receptors is regulated via protein kinase A-mediated phosphorylation. J Neurosci 18:9227-9237.

Pattillo JM, Yazejian B, DiGregorio DA, Vergara JL, Grinnell AD, Meriney SD (2001) Contribution of presynaptic calcium-activated potassium currents to transmitter release regulation in cultured Xenopus nerve-muscle synapses. Neuroscience 102:229-240.

Saint-Amant L, Drapeau P (2003) Whole-cell patch-clamp recordings from identified spinal neurons in the zebrafish embryo. Methods Cell Sci 25:59-64.

Sine SM, Steinbach JH (1984) Agonists block currents through acetylcholine receptor channels. Biophys J 46:277-283.

Sine SM, Claudio T, Sigworth FJ (1990) Activation of Torpedo acetylcholine receptors expressed in mouse fibroblasts. Single channel current kinetics reveal distinct agonist binding affinities. J Gen Physiol 96:395-437.

Steinbach JH (1989) Structural and functional diversity in vertebrate skeletal muscle nicotinic acetylcholine receptors. Annu Rev Physiol 51:353-365.

Van der Kloot W (1988) The kinetics of quantal releases during end-plate currents at the frog neuromuscular junction. J Physiol (Lond) 402:605-626.

Westerfield M, McMurray JV, Eisen JS (1986) Identified motoneurons and their innervation of axial muscles in the zebrafish. J Neurosci 6:2267-2277.

Yazejian B, DiGregorio DA, Vergara JL, Poage RE, Meriney SD, Grinnell AD (1997) Direct measurements of presynaptic calcium and calciumactivated potassium currents regulating neurotransmitter release at cultured Xenopus nerve-muscle synapses. J Neurosci 17:2990-3001. 